\title{
DYNAMIC FEEDBACK OPTIMIZATION OF BIORESOURCE MANAGEMENT IN A POLLUTED ECOSYSTEM
}

\author{
Chung-Min Liao \\ Department of Agricultural Engineering, National Taiwan University, Taipei, Taiwan 10617, \\ Republic of China
}

EI 9806-163 M (Received 26 June 1998; accepted 25 November 1998)

\begin{abstract}
A single state bioresource management model was derived from the viewpoint of control engineering based on a nonlinear and stochastic dynamic system. The model can be used to solve the problem regarding the analysis and management of biological resources in the presence of uncertainty and the implication of uncertainty in decision making. The management of trace $\mathrm{Zn}$ bioaccumulation in aquaculture abalone, Haliotis diversicolor supertexta, is illustrated to show the applicability of established techniques in designing a dynamic feedback control strategy. General results from the engineering analysis are presented in the form of strategies of constant $\mathrm{Zn}$ concentration in molluskan shellfish and of constant control effort and in the form of warning about the instability of the system. Numerical data were obtained which could be used to design a controller minimizing fluctuations about a chosen equilibrium state. Dynamic feedback optimization can serve as a guide to the decision making of sustainable bioresource management in a polluted ecosystem in that the decisions are required to be made in the presence of uncertainty. (C) 1999 Elsevier Science Ltd
\end{abstract}

\section{INTRODUCTION}

In recent years, the management of biological resources in an ecosystem has become increasingly important and is well established as an important scientific and management issue (Edwards et al. 1993). Walters (1986) and Clark (1990) suggested that the analysis and management of biological resources should be focused on uncertainty and the implication of uncertainty in decision making.

There is concern that increased yield from high chemical inputs may not be sustainable over the longterm development in an ecosystem. The development of the concept of sustainable bioresource is a relatively recent response to concerns about degradation of natural resources. Sustainable bioresource promotes environmental, ecological, economic, and social stability and sustainability (Edwards et al. 1993).
Clark (1990), Williams (1996), and Edwards et al. (1993) indicated that the management of bioresources in an ecosystem is a complex process governed by a variety of political, economic, and biological factors. These may include: 1) the dynamics of reproduction and diminution of the resources which are nonlinear, stochastic, and poorly understood; 2) the uncertainty as to resource status, expressed as sampling variation in the monitoring of resources; 3 ) the uncontrollable environmental variations and unpredictability of environmental effects; and 4) the various and controversial methods for implementing regulation.

Decision makers show account for such effects, because management strategies that allow for uncertainties can be different from management strategies that do not take into account the uncertainty effects. 


\begin{tabular}{|c|c|}
\hline \multicolumn{2}{|r|}{ NOTATIONS } \\
\hline$a$ & Stability coefficient \\
\hline$b(t)$ & Biomass in the time $t(\mathrm{mg})$ \\
\hline$B$ & Equilibrium value of biomass $b(t)(\mathrm{mg})$ \\
\hline$c(t)$ & $\begin{array}{l}\text { Pollutant concentration in the biomass at } \\
\text { time } t\left(\mu \mathrm{g} \mathrm{g}^{-1}\right)\end{array}$ \\
\hline$c^{*}(t)$ & Optimal value of $c(t)\left(\mu \mathrm{g} \mathrm{g}^{-1}\right)$ \\
\hline$C$ & Equilibrium value of $c(t)\left(\mu \mathrm{g} \mathrm{g}^{-1}\right)$ \\
\hline$c_{b}(t)$ & $\begin{array}{l}\text { Amount of pollutant accumulated in } \\
\text { contaminated resource at time } t\left(\mu \mathrm{g} \mathrm{g}^{-1}\right)\end{array}$ \\
\hline$C_{b}$ & Equilibrium value of $c_{b}(t)\left(\mu \mathrm{g} \mathrm{g}^{-1}\right)$ \\
\hline$d(t)$ & Diminution rate at time $t$ (dimensionless) \\
\hline$f(t)$ & Control effort at time $t$ (dime \\
\hline$G(b(t))$ & $\begin{array}{l}\text { Biomass production dynamic function } \\
\text { (number biota) }\end{array}$ \\
\hline$h(t)$ & Harvest rate at time $t$ (dimensionless) \\
\hline$J_{T}$ & $\begin{array}{l}\text { Performance index of control over an } \\
\text { extended } T \text { years }\end{array}$ \\
\hline$m$ & ion rate (dimensionless) \\
\hline$M$ & Equilibrium $\mathbf{v}$ \\
\hline$L N$ & Log-normal distribution fur \\
\hline$q$ & $\begin{array}{l}\text { Relative diminution capacit } \\
\text { (dimensionless) }\end{array}$ \\
\hline$s(t)$ & Surplus rate a \\
\hline$w_{b}$ & Weight of bio \\
\hline$x(t)$ & $\begin{array}{l}\text { Stock numbe } \\
\text { the system at }\end{array}$ \\
\hline$X$ & Equilibrium value of $x(t)$ (di \\
\hline$x_{c}(t)$ & $\begin{array}{l}\text { Number resource contami } \\
\text { pollutant (number biota) }\end{array}$ \\
\hline$X_{M A X}$ & Maximum value of $X$ (number biota) \\
\hline$X_{M S Y}$ & $\begin{array}{l}\text { Maximum sustainable yield of } X \text { (number } \\
\text { biota) }\end{array}$ \\
\hline$\hat{x}(t / t)$ & Estimate of $x(t)$ (number biota) \\
\hline$y(t)$ & Mea \\
\hline$\Delta c(t)$ & for $c(t)$ \\
\hline$\Delta c^{*}(t)$ & Suboptimal feedback control variable \\
\hline$\Delta f(t)$ & Perturbation term for $f(t)$ \\
\hline$\xi(t), \zeta(t$ & $\begin{array}{l}\text { Random variables for } G(b(t)) \text { and } y(t) \text {, } \\
\text { respectively }\end{array}$ \\
\hline & Constant weighting parameter \\
\hline & Fitting parameters of $G(b(t))$ \\
\hline$\mu_{\xi} \sigma_{\xi}^{2}$ & Mean and variance for $\xi(t)$ \\
\hline$\mu_{\sigma}, \sigma_{\zeta}^{2}$ & Mean and variance for $\zeta(t)$ \\
\hline
\end{tabular}

Efforts to clean up a polluted ecosystem have lead to many regulations on the amount of pollutant allowed in the environment. The establishment of regulations depends on the type of behavior desired in the system. Generally, the objective is to prevent the bioresource from being contaminated and maintain function of the ecosystcm.

The problems of managing bioresources in polluted ecosystems could be formulated and solved in terms of the concept of control engineering (Liao and Lin 1997; Thomas et al. 1996). The controlled object, for example, could be the resources from which control effort $(f)$ balances resource stock $(x)$ and concentration of pollutant in the biomass of the resources $(c)$. The relationship between these variables is governed by uncertain internal dynamics and production processes of the resources as well as by exogenous disturbances. The values of $c$ and $x$ are not precisely known, but more or less accurate estimates $\hat{c}$ and $\hat{x}$ are used as a basic for management decisions and represented by the feedback controller. Figure 1 shows the block diagram representing bioresource management in a polluted ecosystem as a problem in feedback control in that the control is achieved in the presence of uncertainty about the relationships governing variables and about the value of variables.

Clark (1990), Goh (1980), Mangel (1985), and Walters (1986) noted that a truly optimal feedback control law for idealized mathematical models must account for how the uncertainty will affect future states of the controlled process. Many researchers such as Williams (1996), Tiwari et al. (1996), Edwards et al. (1993), and Liao and Lin (1997) revealed that the problem of polluted ecosystem management is too nonlinear to be well controlled by a linear feedback controller coupled with a mathematical model of resource dynamics.

In a polluted ecosystem, the significant features to be modeled in bioresource management could be subjected to: 1) nonlinearity in the underlying dynamics of the resources; 2) random fluctuation in the production capacity of the resources; 3 ) a management objective of eliminating fluctuations in both $c$ and $f$ to maintain equilibrium; 4) uncertainty about current resource stock; and 5) uncontrollable and unpredictable environmental variations.

Because of nonlinearity and uncertainty in even a simple model, general procedures for designing linear controllers to match linear controlled processes are not appropriate. The controller design can only be suboptimal, and is obtained in the form of a suboptimal state estimator. The effectiveness of the resulting feedback controller was investigated by simulating its performance when controlling the simple mathematical model as well as a stochastic resource dynamics in a polluted ecosystem. 


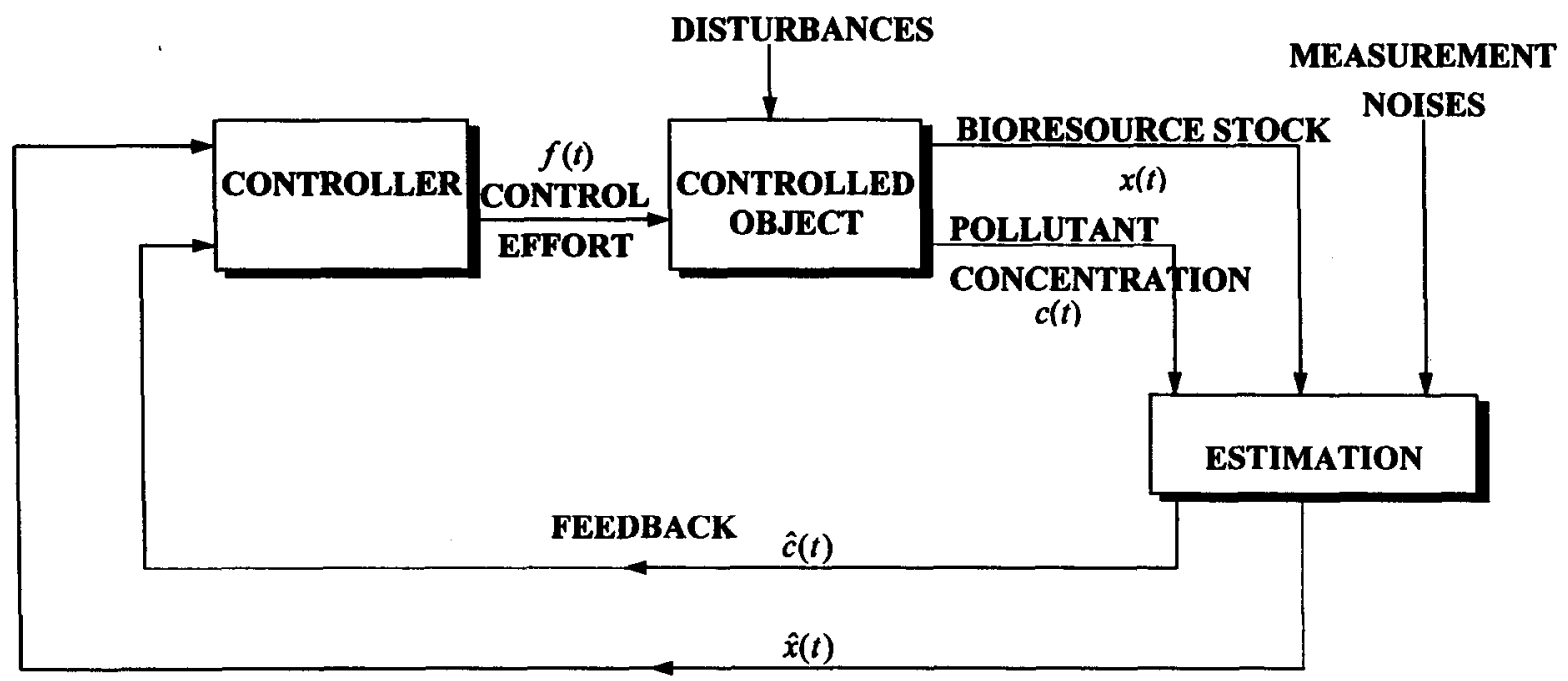

Fig. 1. Block diagram representing bioresource management in a polluted ecosystem as a problem in feedback control.

The three variables of control effort $f$, resource stock $x$, and pollutant concentration in the biomass $c$ can easily be manipulated so that $c$ rather than $f$ is regarded as the actuating signal. This corresponds to management by setting resource exposure limits as is common practice by giving a maximum allowable dose, and is the basis of the controller design.

Furthermore, itwishes to force $\lim _{t \rightarrow \infty} c(t)=L$ where $L$ is considered an environmental quality limit determining the amount of pollutant allowed in the ecosystem. In an ideal situation, it is preferable to have the tuning signal to be able to drive $c(t)$ to $L$. From a feedback control system point of view, the information of the amount of pollutant accumulated in the biomass of the resources has to feed back to the controller in order for $c(t)$ to reach the desired value $L$.

The aim of this paper is to study the dynamic feedback control of a single bioresource in a polluted ecosystem where the input of pollution is controlled. The management of one particular bioresource is considered. This paper will discuss how uncertainty about current states of the controlled process, e.g., current resource stock, affect the design of a feedback controller and performance of the resulting system.

\section{MODEL STRUCTURE}

\section{Model development}

Both Emlen and Pikitch (1989) and Barnthouse (1993) noted that age/stage-structured population models, i.e., models in which all organisms belonging to a population are classified into groups according to age, size, and reproductive status, have a long history of application in natural resource management. These models are readily available quantitative methods for assessing the risks of toxic chemicals to resources.

Variables used in the model to characterize the management of bioresource in an ecosystem are: $x(t)=$ state variable, the stock number of bioresource available to the system at time $t ; c(t)=$ observed (actuating) variable, the amount of pollutant in the biomass of the resource at time $t\left(\mu \mathrm{g} \mathrm{g}^{-1}\right)$; and $f(t)=$ control variable, the control effort during time $t$ (a dimensionless parameter per year).

The basic mathematical model of the resource dynamics for a single bioresource without considering age distribution could be expressed by a first-order stochastic difference equation,

$$
x(t+I)=\xi(t) G(b(t))+x(t) s(t),
$$

where $\xi(t)$ is a random variable. Hines and Montgomery (1982) suggested that random variables characterizing uncertainty about resource production dynamics could be assumed to have log-normal distributions. Therefore, $\xi(t)$ is assumed to have a lognormal distribution with mean $\mu_{\xi}$ and variance $\sigma_{\xi}^{2}$ as $\xi(t) \sim L N\left(\mu_{\xi}, \sigma_{\xi}^{2}\right)$ in that,

$$
L N\left(\mu_{\xi} \sigma_{\xi}^{2}\right)=\frac{1}{\sqrt{2 \pi} \xi 1 n \sigma_{\xi}} \exp \left(-\frac{\left(\ln \xi-\ln \mu_{\xi}\right)^{2}}{2\left(\ln \sigma_{\xi}\right)^{2}}\right) .
$$


$G(b(t))$ in Eq. 1a is a nonlinear resource production dynamic function of the biomass $b(t)$ in that $b(t)=x(t) w_{b}$ where $w_{b}$ is the weight of biomass of resource (mg), and $s(t)$ is a surplus rate which depends on the control effort $f(t)$.

The seemingly random dynamics of Eq. 1 pose serious problems from a modeling point of view. What modeling can do, however, is to point to how sensitive the resource dynamics can be to the changes in environmental parameters, the estimation of which is often difficult and usually important.

The resource production function $G(b(t))$ is perhaps the most arbitrary feature of the mathematical model because little is known, neither from theory nor from observation, about what actually determines bioresource production dynamics. All the production dynamics models which have been taken from the ecological literature are of the form $G(b(t))$ and all have been used in modeling real situations. The bioresource production function used in this model then is chosen from a well-known expression as (Murray 1989):

$$
G(b(t))=\frac{\alpha b(t)}{1+(\gamma b(t))^{\beta}},
$$

where $\alpha, \beta$, and $\gamma$ are fitting parameters which can be matched to the observed data.

According to Anderson (1975) and Williams (1996) on the management of renewable biotic resources, the surplus rate $s(t)$ is considered to depend on a harvest rate,

$$
s(t)=s_{o}(1-h(t)),
$$

where $s_{o}$ is a surplus rate in the absence of hunting, and $h(t)$ is a harvest rate. In the present model, the harvest rate $h(t)$ could be replaced by a diminution rate $d(t)$ accounting for all possible diminution causes. The diminution rate $d(t)$ is assumed to depend on the function of control effort $f(t)$, thus the surplus rate in Eq. 3 could be rewritten as:

$$
s(t)=s_{o}(1-d(t))=s_{o}\left(1-\left(m+q f(t)^{-1}\right)\right),
$$

where $m$ is the natural diminution accounting for the resource diminished by natural causes during the year $t$; and $q$ is a dimensionless parameter representing a rclative diminution capacity of resources which are contaminated in a polluted environment, where the input of pollutant is controlled under the control effort $f(t)$.

The number of resources which diminished is $(1-s(t)) x(t)$, of which the proportion contaminated is $q f(t)^{-1} /\left(q f(t)^{-1}+m\right)$. Thus, the concentration of pollutant in the biomass of the resource $(c(t))$ in a polluted ecosystem is given as:

$$
c(t)=c_{b}(t)\left(\frac{q f(t)^{-1}}{q f(t)^{-1}+m}\right)(1-s(t)) x(t),
$$

where $c_{b}(t)$ is the amount of pollutant accumulated in a contaminated resource at time $t\left(\mu \mathrm{g} \mathrm{g}^{-1}\right)$, while the number of resources contaminated by the pollutant is defined as:

$$
x_{c}(t)=\left(\frac{q f(t)^{-1}}{q f(t)^{-1}+m}\right)(1-s(t)) x(t) .
$$

Nonlinearity in the model arises partly because of the nonlinear resource production function $G(b(t))$ in Eq. 2 and partly because of the nonlinear control effort $f(t)$ and contaminated biomass function $c(t)$ in Eq. 5 .

\section{Management objective}

The management objective is to maintain the bioresources in a state of dynamic equilibrium such that control effort $f(t)$ balances bioresource production capacity $G(b(t))$. Random fluctuations in resource production dynamics are exercised to minimize the resulting fluctuations in both $c(t)$ and $f(t)$.

The sum of an equilibrium and a perturbation forms a controlled object as:

$$
\begin{aligned}
& c(t)=C+\Delta c(t) \\
& f(t)=F+\Delta f(t),
\end{aligned}
$$

where $C$ and $F$ are equilibrium values, while $\Delta c(t)$ and $\Delta f(t)$ give the perturbation terms, respectively, for $c(t)$ and $f(t)$.

The performance index of control over an extended $T$ years can then be measured by a quadratic cost function based on the averaged sum of the squared error as: 
Table 1. Overall model equations describing the dynamic feedback control system of bioresource management in a polluted ecosystem in which $x(t)=$ state variable, $f(t)=$ control variable, $c(t)=$ observed (actuating) variable, and $y(t)=$ output variable.

$$
\begin{aligned}
& \text { State equation: } x(t+I)=\xi(t) G(b(t))+x(t) s(t), \xi(t) \sim L N\left(\mu_{\xi} \sigma_{\xi}^{2}\right) \\
& \text { Bioresource production function: } G(b(t))=\frac{\alpha b(t)}{1+(\gamma b(t))^{\beta}} \\
& \text { Surplus rate: } s(t)=s_{o}\left(I-\left(m+q f(t)^{-t}\right)\right) \\
& \text { Pollutant in biomass: } c(t)=c_{b}(t)\left(\frac{q f(t)^{-1}}{q f(t)^{-1}+m}\right)(1-s(t)) x(t) \\
& \text { Controlled object: } c(t)=C+\Delta c(t), f(t)=F+\Delta f(t) \\
& \text { Objective function: } J_{T}=\frac{1}{T} \sum_{t=0}^{T} \lambda\left(\frac{\Delta f(t)}{F}\right)^{2}+\mu\left(\frac{\Delta c(t)}{C}\right)^{2} \\
& \text { Output equation: } y(t)=\zeta(t) x(t), \zeta(t)-L N\left(\mu_{\sigma}, \sigma_{\zeta}^{2}\right)
\end{aligned}
$$

$$
\begin{gathered}
J_{T}=\frac{1}{T} \sum_{t=0}^{T} \lambda\left(\frac{f(t)}{F}-1\right)^{2}+\mu\left(\frac{c(t)}{C}-1\right)^{2}, \\
0 \leq \lambda \leq 1,0 \leq \mu \leq 1,
\end{gathered}
$$

where $\lambda$ and $\mu$ are constant weighting parameters. Particularly $\lambda+\mu=1$ is considered to reflect the relative importance of fluctuations in $c(t)$ against fluctuations in $f(t)$.

\section{Feedback information}

Practically, it is not always possible to have current values of state variable $x(t)$ available for feedback. What is available may be imperfect estimates arising from virtual resource dynamics analysis. Rather than reconstructing the state variables via a Kalman filter or some form of a state estimator, it is preferable to generate the control variables by taking linear combinations of the available output variables.

The resulting uncertainty about current contaminated resource stock is thus modeled by assuming that a set of noisy measurements $y(t)$ is available to the system as:

$$
y(t)=\zeta(t) x(t), \zeta(t) \sim L N\left(\mu_{\zeta}, \sigma_{\zeta}^{2}\right)
$$

where $\zeta(t)$ is the measurement noise of an independent random variable having log-normal distribution with mean $\mu_{\zeta}$ and variances $\sigma_{\zeta}^{2}$.
Delays in making and implementing management decisions are modeled in the form of one-year deadtime before the measurements $y(t)$ become available. The management decision, say $u(t)$ affecting control effort in year $t$, thus has information structure characterized by the notation, $u(t)=u(\bar{y}(t-1), \bar{u}(t-1))$, where $\bar{y}(t-1)$ represents a sequence of past data up to and including $y(t-1)$ (Aoki 1976; Jocobs 1993). The overall model equations describing the dynamic feedback optimization of bioresource management in a polluted ecosystem are summarized in Table 1.

\section{CONTROLLER DESIGN}

\section{Suboptimal feedback controller}

The overall feedback control scheme is to minimize fluctuations about a desired state of the resource stock in a polluted ecosystem. This desired state is specified by giving a desired equilibrium value for either resource stock $X$ or control effort $F$. These equilibrium values depend on each other. The controller output or the actuating signal is $c(t)$.

Equation 5 can be approximately expressed as $s_{o}$ taken to be unity in Eq. 3:

$$
c(\mathrm{t}) \approx c_{b}(t) q f(t)^{-l} x(t) .
$$

For a given controlled process in Eq. 7, a suboptimal feedback control variable $\Delta c^{*}(t)$ is desired that will 
minimize the quadratic cost function (Eq. 8). The solution of the suboptimal feedback control variable $\Delta c^{*}(t)$ could be obtained by eliminating $f(t)$ based on Eq. 10:

$$
\Delta c^{*}(t)=\frac{\left(C \frac{\Delta x(t)}{X}\right)}{1+\left(\frac{\mu}{1-\mu}\right)\left(1+\frac{\Delta x(t)}{X}\right)^{2}}
$$

Equation 11 is a basic nonlinear feedback control law throughout the model. When $\mu=0$, Eq. 11 becomes $\Delta c^{*} / \Delta x=C / X$ so that $c(t) / x(t)$ remains constant and the control effort $f(t)$ depends only on $c_{b}(t)$ (Eq. 10). If $c_{b}(t)$ keeps at a desired equilibrium value (say, $C_{b}$ ), $f(t)$ remains constant: a constant effort control law. This control law is perhaps the most obvious suboptimal linear feedback control which could have been suggested without the above nonlinear derivation. When $\mu=1$, Eq. 11 becomes $\Delta c^{*}(t)=0$ so that $c^{*}(t)=C$ : a constant concentration control law which is not a feedback control.

The nonlinear characteristics of the basic feedback control law (Eq. 11) are illustrated in Fig. 2, where normalized $c(t) / C$ is plotted against normalized $x(t) / X$ for different tuning parameters of $\mu$ ranged from 0 to 1 . Figure 2 shows at intermediate values of the tuning parameters $(0.1 \sim 0.9)$, the control law lies between the extremes of constant control effort and constant pollutant concentration in the biomass. For values of pollutant concentration in the biomass less than the target equilibrium values $(c / C<1)$, the control law is appropriately implemented to the management of resource in polluted ecosystems.

In order to implement Eq. 11, information is needed about the target equilibrium values of $C$ and $X$, which are assumed to be given as a specification of desired state, and about the current stock number of the resource $x(t)$ in that $x(t)-X=\Delta x(t)$. Because of the deadtimc in decision making, the most up-to-date feedback information about the species population $x(t)$ will be delayed measurements from Eq. 9. Therefore, the best available estimate of $x(t-1)$, i.e., $\hat{x}(t-1 \mid t)$, could be replaced by $y(t-1)$.

A corresponding estimate of $x(t)$ is then obtainable by using Eq. 1 , and assuming that $\xi(t-1)$ took its expected value of unity to make a form of a certainty equivalent prediction (Polderman and Willems 1998): $\hat{x}(t \mid t)=G\left(\hat{x}(t-1 \mid t) w_{b}\right)+\hat{x}(t-1 \mid t) s(t-1)$.

Replacing $\hat{x}(t-1 \mid t)$ by $y(t-1)$ and using the approximation of $c(t)$ in Eq. 10, Eq. 12 can be rewritten as:

$$
\hat{x}(t \mid t) \approx G\left(y(t-1) w_{b}\right)+(1-m) y(t-1)-\frac{c(t-1)}{c_{b}(t-1)} .
$$

The controlled object $c^{*}(t)=C+\Delta c^{*}(t)$ to be implemented at time $t$ can then be computed by using $\hat{x}(t \mid t)-X$ from Eq. 13 to replace $\Delta x(\mathrm{t})$ in Eq. 11 .

The approximate equilibrium value $X$ is found by substituting $\xi(t)=1$ into Eq. 1 with a given equilibrium value $F$ to yield an implicit equation:

$$
X=G\left(X w_{b}\right)+X\left(1-m-q F^{1}\right),
$$

and gives a final expression of $X$ :

$$
X=\frac{m+q F^{-1}-\alpha w_{b}}{\gamma w_{b}\left(m+q F^{-1}\right)} .
$$

Table 2 lists the approximated version of the suboptimal dynamic feedback control model based on a certainty equivalent prediction. Figure 3 illustrates the block diagram representing the structure of the dynamic feedback control system for bioresource management in a polluted ecosystem.

\section{Stability analysis}

The concept of stability is extremely important since every workable system must be designed to be stable. It is not easy to analyze the stability of a system which is controlled by the nonlinear suboptimal controller of Eq. 11. Stability, however, can be obtained from analysis of a deterministic reduced order feedback system based on Eqs. 1 and 10 with $\xi(t)=1$ and an equilibrium value $C_{b}$ for $c_{b}(t)$ :

$$
x(t+1)=G\left(w_{b} x(t)\right)+(1-m) x(t)-\frac{c(t)}{C_{b}} .
$$

Equation 15 is also controlled by the nonlinear control law (Eq. 11). 


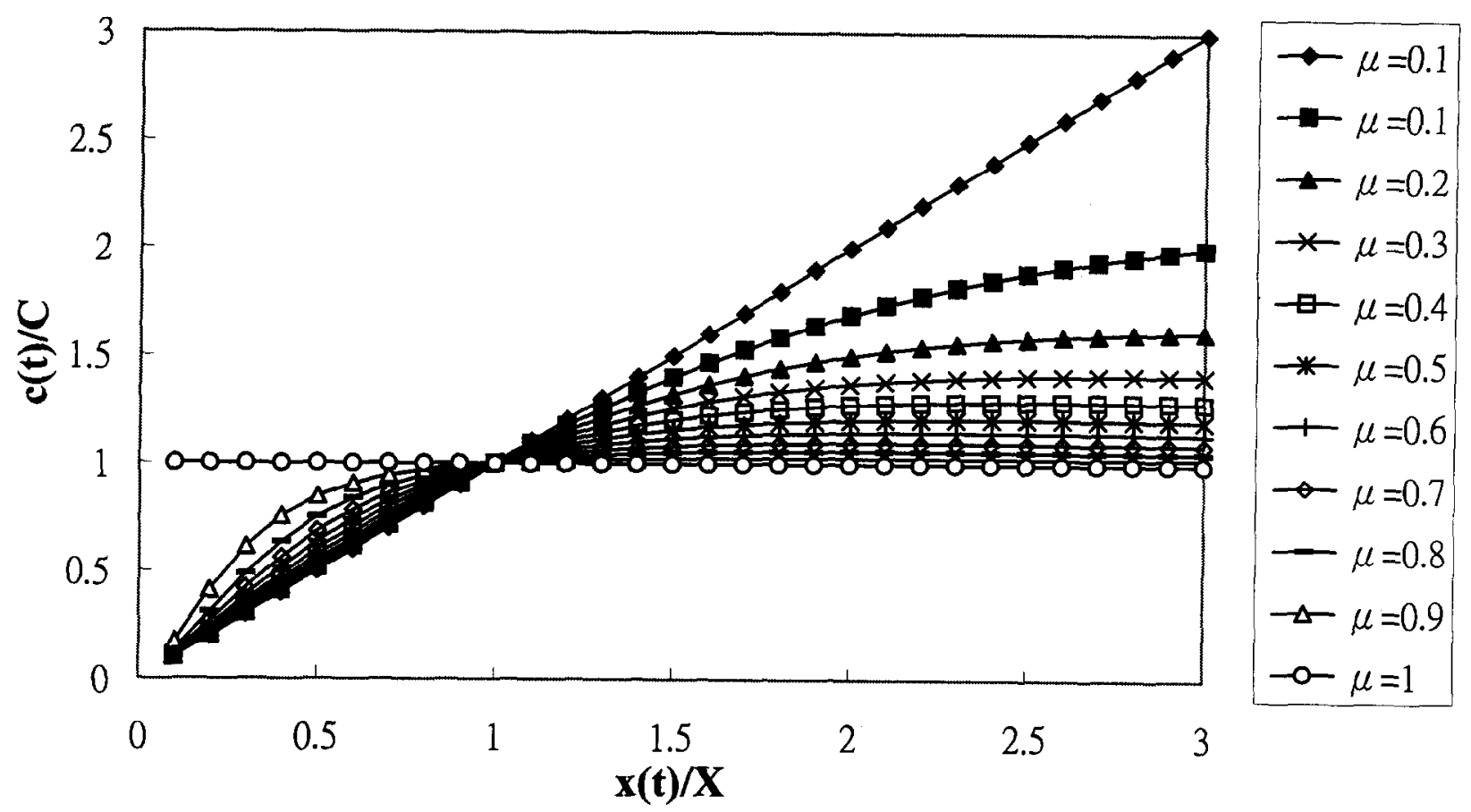

Fig. 2. The nonlinear characteristics of the basic feedback control law used in the model in which $\mu=0$ : a constant effort control law, and $\mu=1$ : a constant concentration law.

Table 2. Approximated version of the suboptimal dynamic feedback control model based on the certainty equivalent prediction in which $x(t)=$ state variable, $c^{*}(t)=$ control variable, and $y(t)=$ output variable.

State equation: $\quad \hat{x}(t \mid t)=G\left(y(t-1) w_{b}\right)+(1-m) y(t-1)-\frac{c(t-1)}{c_{b}(t-1)}$

Controlled object: $\quad c^{*}(t)=C+\Delta c^{*}(t)$

Nonlinear feedback control law: $\Delta c *(t)=\frac{\left(C \frac{\Delta x(t)}{X}\right)}{1+\left(\frac{\mu}{1-\mu}\right)\left(1+\frac{\Delta x(t)}{X}\right)^{2}}$

where: $\quad X=\frac{m+q F^{-1}-a w_{b}}{g w_{b}\left(m+q F^{-1}\right)}, D x(t)=\hat{x}(t \mid t)-X$

Output equation: $\quad y(t)=\zeta(t) \hat{x}(t \mid t), \zeta(t) \sim L N\left(\mu_{\zeta}, \sigma_{\zeta}{ }^{2}\right)$ 


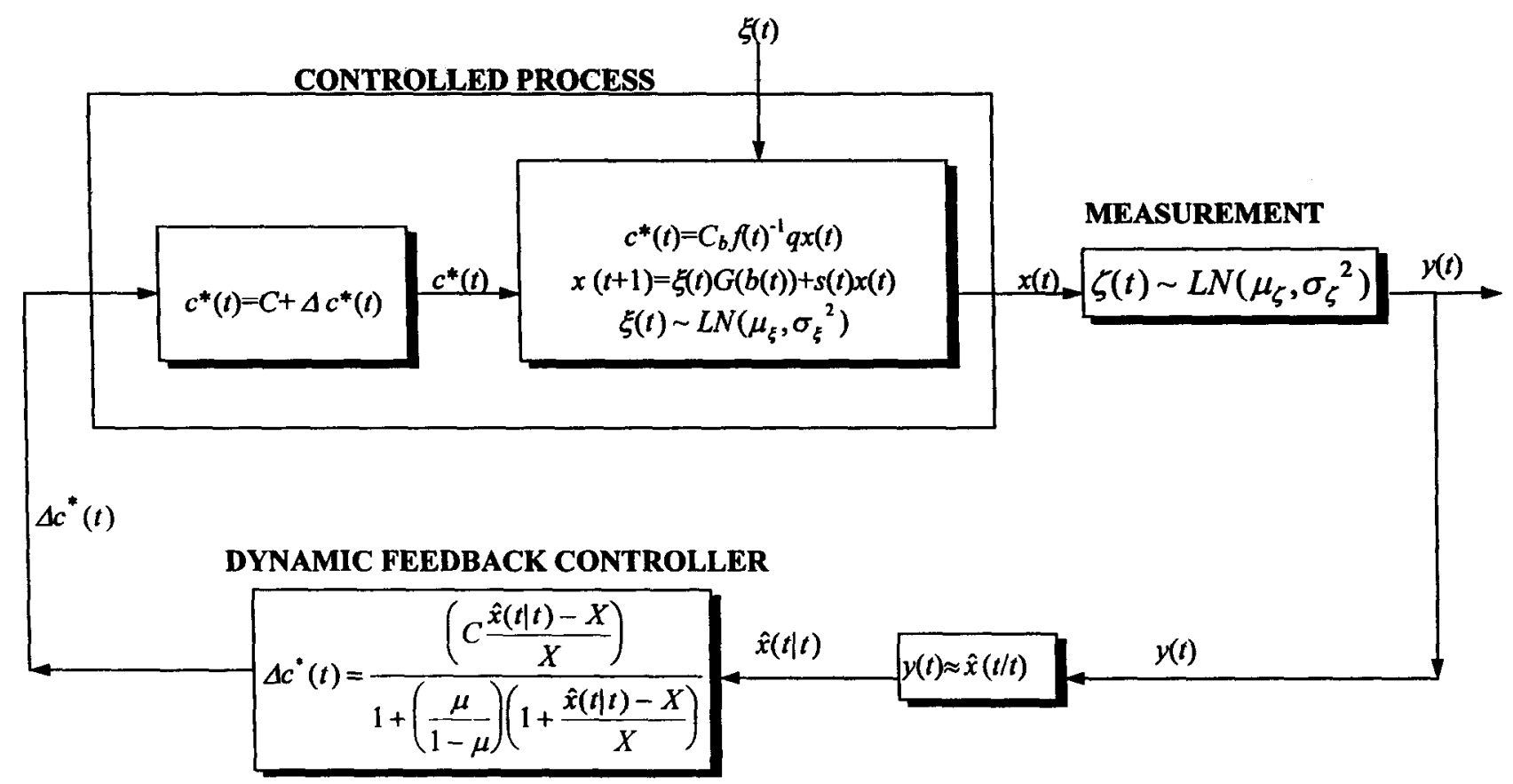

Fig. 3. Block diagram representing the structure of the dynamic feedback control system for bioresource management in a polluted ecosystem.

Table 3. Linearized version of the model: a deterministic reduced order feedback control model of bioresource management in which $x(t)=$ state variable, $\Delta c(t)=$ control variable, and $y(t)=$ output variable.

$$
\begin{aligned}
& \text { State equation: } x(t+1)=G\left(w_{b} x(t)\right)+(1-m) x(t)-\frac{c(t)}{C_{b}} \\
& \text { Controlled objective: } c(t)=C+\Delta c(t) \\
& \text { Nonlinear feedback control law: } \Delta c(t)=(1-\mu) \frac{C}{X} \Delta x(t) \\
& \text { where, } C=C_{b}\left(G\left(X w_{b}\right)-m X\right), X=\frac{m+q F^{-1}-\alpha w_{b}}{\gamma w_{b}\left(m+q F^{-1}\right)}, \Delta x(t)=X-x(t) \\
& \text { Output equation: } y(t)=\zeta(t) x(t), \zeta(t) \sim L N\left(\mu_{\zeta}, \sigma_{\zeta}^{2}\right)
\end{aligned}
$$

The linearized equation of the feedback control law (Eq. 11) is:

$$
\Delta c(t)=(1-\mu) \frac{C}{X} \Delta x(t),
$$

in which the equilibrium value $C$, based on $\mathrm{Eq} .10$, gives:

$$
C=C_{b}(G(B)-m X),
$$

where $B=X w_{b}$ is the equilibrium value of biomass $b(t)$.
Table 3 gives the linearized version of the feedback control model of bioresource management.

Stability analysis uses linearized equations which are assumed to govern small fluctuations about the target equilibrium point. Combining Eqs. 15 and 16 with relations $x(t)=X+\Delta x(t)$ yields:

$$
\begin{aligned}
X+\Delta x(t+1)= & G\left(w_{b}(X+\Delta x(t))\right)+(1-m)(X+\Delta x(t)) \\
& -\frac{C}{C_{b}}\left(1+(1-\mu) \frac{\Delta x(t)}{X}\right)
\end{aligned}
$$


Table 4. Numerical values used in the model simulation.

\begin{tabular}{lcccc}
\hline Age group & $\begin{array}{c}\text { Abalone weight } \\
\mathrm{w}_{\mathrm{b}}(\mathrm{g})\end{array}$ & $\begin{array}{c}\text { Zn concen. in abalone } \\
\mathrm{C}_{\mathrm{b}}\left(\mu \mathrm{g} \mathrm{g}^{-1}\right)\end{array}$ & $\begin{array}{c}\text { Relative lost capacity } \\
\mathrm{q}_{\mathrm{i}}\end{array}$ & $\begin{array}{c}\text { Natural diminution rate } \\
\mathrm{m}_{\mathrm{i}}\end{array}$ \\
\hline 1 & 20.34 & 13.1 & 0.5 & 0.15 \\
2 & 49.78 & 24.8 & 1.0 & 0.15 \\
3 & 134.67 & 28.3 & 1.0 & 0.15 \\
4 & 223.39 & 34.3 & 1.0 & 0.15 \\
5 & 245.52 & 37.3 & 1.0 & 0.15 \\
6 & 346.13 & 45.5 & 1.0 & 0.15 \\
7 & 385.65 & 49.7 & 1.0 & 0.15 \\
8 & 423.35 & 50.8 & 1.0 & 0.15 \\
9 & 531.30 & 53.9 & 1.0 & 0.15 \\
$10+$ & 531.30 & 57.3 & 1.0 & 0.15 \\
\hline
\end{tabular}

$\bar{w}_{b}=283.54 \mathrm{~g}, \bar{q}=0.95, \bar{m}=0.15$.

The fluctuation $\Delta x$ is governed by a first-order linear difference equation:

$$
\Delta x(t+1)=a \Delta x(t)
$$

Comparing Eqs. 18 and 19, the stability coefficient $a$ is obtained to reflect the dynamic characteristics:

$$
a=1-\mu m+w_{b} G^{\prime}(B)-(1-\mu) \frac{G(B)}{X}>0,
$$

where $G^{\prime}(B)$ is the first derivative of the resource production dynamic function in Eq. 2:

$$
G^{\prime}(B)=\alpha \frac{1+(1-\beta)(\gamma B)^{\beta}}{\left.1+(\gamma B)^{\beta}\right)^{2}}
$$

In view of Eq. 20 , for an equilibrium at $\Delta x(t)=0$, the only cases in which the solution approaches and remains at equilibrium are the cases where $a<1$, i.e., the model is stable if $a<1$.

\section{A NUMERICAL SIMULATION}

A dynamic feedback control of bioresource management in a polluted ecosystem is illustrated in terms of renewable biotic resources. A numerical simulation is presented here to get insight into the whole concept introduced.
Conory et al. (1996) and Ritterhoff and Zauke (1997) indicated that gastropod and bivalve mollusks represent the most important source of heavy metals to human populations. Elevated heavy metal concentrations in molluskan shellfish have often been reported in aquatic ecosystems far from direct sources of heavy metal pollution. The health risk associated with heavy metal intoxication to humans together with the global heavy metal contamination of polluted aquatic ecosystems necessitates the development of a management model for regulating heavy metal bioaccumulation in molluskan shellfish.

The aquaculture of abalone, Haliotis diversicolor supertexta is considered one of the most important economic aquatic products in Taiwan. Moreover, $H$. diversicolor supertexta is appreciated for its delicacy and high market value. The aquaculture of $H$. diversicolor supertexta, therefore, is a promising business in Taiwan. Thus, $H$. diversicolor supertexta is chosen as a harvest bioresource organism in the present work for studying the management and design of a dynamic feedback control strategy for the mollusk Zn-bioaccumulation in a polluted ecosystem. Numerical data given in Table 4 were used as a basic database for all the present work. Numerical values for the parameters in this model were calculated from Table 4 in that part of the numerical values were adapted from Lin and Liao (1999) regarding the experiment results of uptake and depuration kinetics and bioaccumulation factors of $\mathrm{Zn}$ in $H$. diversicolor supertexta.

Input parameters for the model simulation are as follows: 


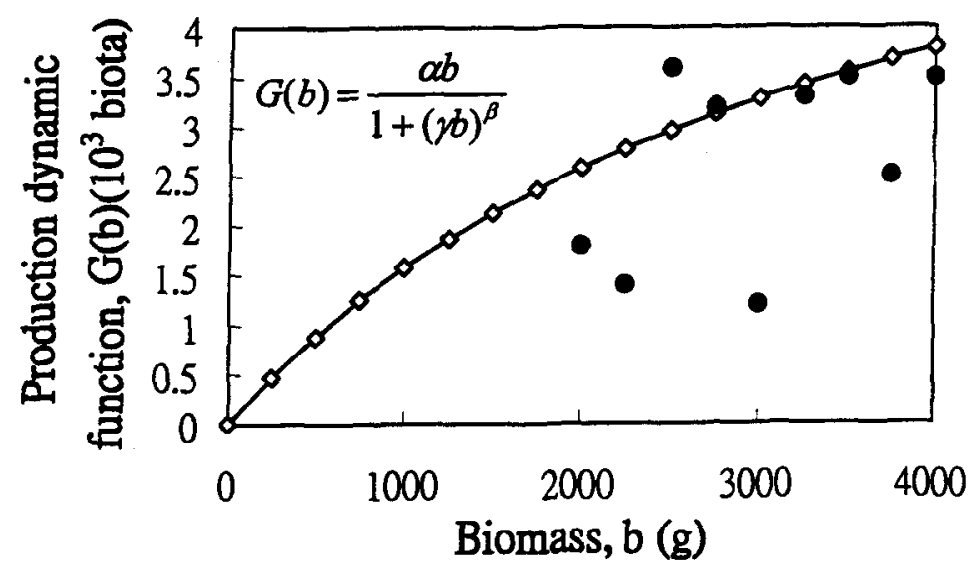

Fig. 4. The resource production dynamic function $G(b)$ plotted against biomass $b$ in which the observed data are shown as dots. The resulting values are: $\alpha=0.00202 \mathrm{biota} / \mathrm{g}, \beta=1$, and $\gamma=(3542.7 \mathrm{~g})^{-1}$.

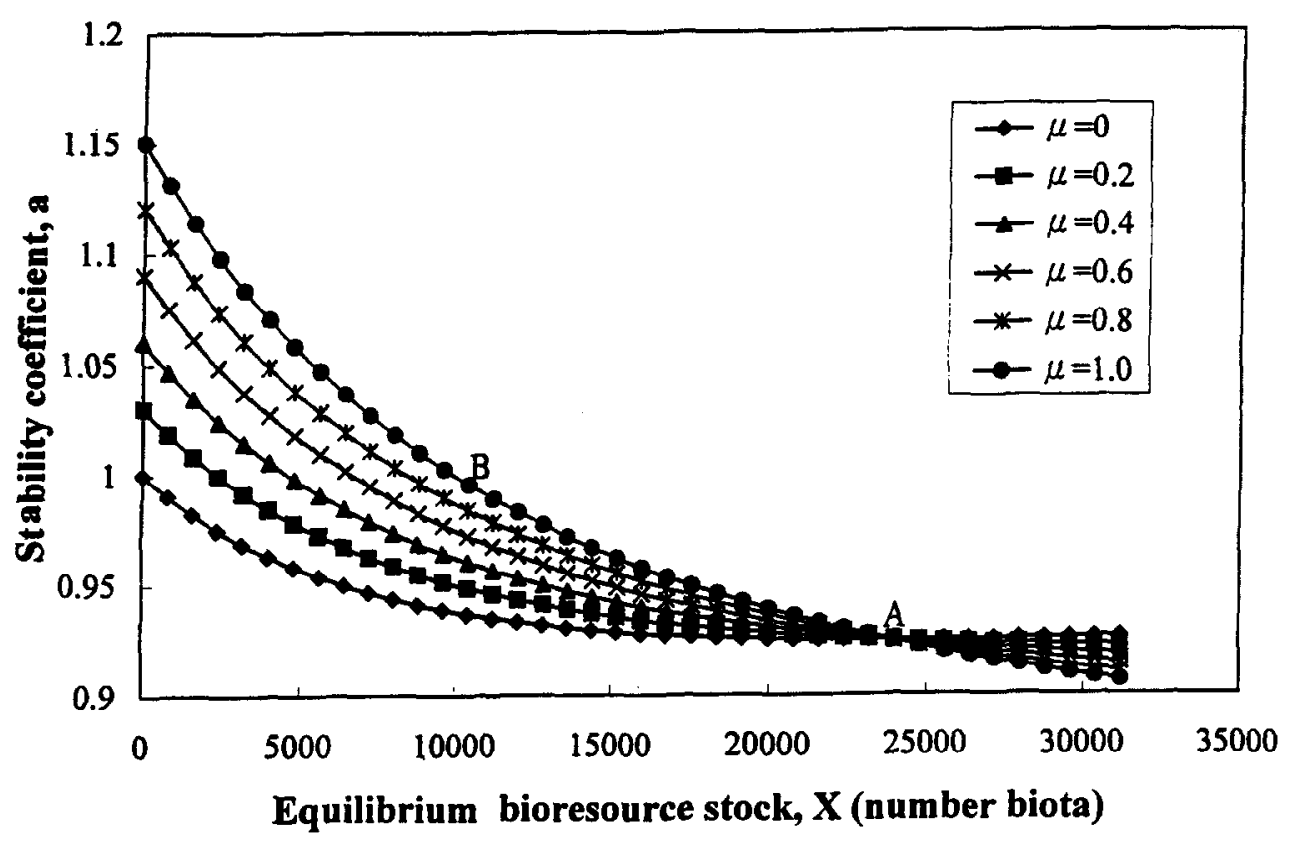

Fig. 5. Stability coefficient $a$ plotted against equilibrium bioresource stock $X$ in the deterministic reduced order feedback system.

1) $\alpha, \beta$, and $\gamma$ in the resource production dynamic function $G(b): \alpha, \beta$, and $\gamma$ can be obtained by matching the production dynamic function in Eq. 2 to an observed data such as is shown in Fig. 4. Figure 4 shows $G(b)$ plotted against biomass $b$. The resulting values are: $\alpha=0.00202 \mathrm{biota} / \mathrm{g}, \beta=1$, and $\gamma=(3542.7 \mathrm{~g})^{-1}$.

2) Values of the three other parameters: the natural diminution rate $m$, average relative lost capacity of bioresource $q$, and average weight of biomass $w_{b}$. The resulting values calculated based on Table 4 are: $m=0.15, q=0.95$, and $w_{b}=283.54 \mathrm{~g}$.
3) Free parameters were the equilibrium state characterized by $F, X$, or $C$; and the coefficient $\mu$ in the control law (Eq. 11) which served as a tuning parameter.

4) The variance of $G(b)$ noise was held constant at $\sigma_{\xi}^{2}$ $=0.25$. In most of the bioresource management literature, it is often assumed that $\mu_{\xi}=0$. In this present work, no such restrictive assumption was made and the. mean and variance for log-normal distribution are specified independently. Here $\mu_{\xi}=\mu_{\zeta}=1$ was chosen in the simulation. 
The numerical scheme used to implement the simulations was done in double precision with FORTRAN 77. The first step was to verify the stability of the model. When $\beta=1$, the stability coefficient $a$ in Eq. 20 becomes:

$$
a=1-\mu m+\frac{\alpha w_{b}}{\left(1+X w_{b} \gamma\right)^{2}}-(1-\mu) \frac{\alpha w_{b}}{1+\gamma X w_{b}}
$$

Figure 5 shows how values of $a$ vary over the range of values in $X$ and $\mu$ for the model. The maximum value of $X$ (point $A$ in Fig. 5, i.e., $X_{M A X} \approx 24000$ number of biota) is achieved with zero $\mathrm{Zn}$ concentration in Haliotis diversicolor supertexta $(c(t)=0)$. The corresponding minimum stability coefficient $(a \approx 0.925)$ is then determined only by the $G(b)$ and the natural diminution rate $m$. It is unacceptable for values of $a \geq 1$ because it will indicate unstable performance.

Figure 5 shows that large weighting $\mu$ on the cost of fluctuations in $c$ results in low equilibrium stock number $X$. It can be shown that the intersection $A$ in Fig. 5, between the $\mu=1$ (constant $\mathrm{Zn}$ concentration in Haliotis diversicolor supertexta) line and the stability boundary of $a=1$ is at a point (point $B$ ) of value about 11500 number of biota. In a specific term, point $B$ can be referred to as a maximum sustainable yield (Murray 1989 ), i.e., $X_{M S Y} \approx 11500$ number of biota.

Figure 5 also indicates that, at reasonably low levels of bioresource stocks, the optimal value of $\mu$ is closer to unity: a non-feedback control (i.e., a constant pollutant concentration in the biomass). However, if a level is attempted above a certain high value (say, 24000 number biota), $\mu$ approaches zero and a feedback control is required (i.e., a constant control effort).

The stability analysis thus confirms that attempts to regulate control effort to a equilibrium value $F$ are likely to introduce unacceptable instability. This might be avoided by giving a constant control effort controller to reduce $\mu$ towards zero. The constant effort control, however, is sensitive to measurement noise in Eq. 9. A bioresource management in a polluted ecosystem shows conflicting requirements between what is needed for steady-state stability and what is needed for noise rejection.

Equations 1 and 5 were simulated with a single measurement noise of the population size given in Eq. 9 and having noise variance $\sigma_{\zeta}^{2}=0.25$. Feedback control was simulated in the form of $\mathrm{Zn}$ concentration in Haliotis diversicolor supertexta as $c(t)=C+\Delta c(t)$ with
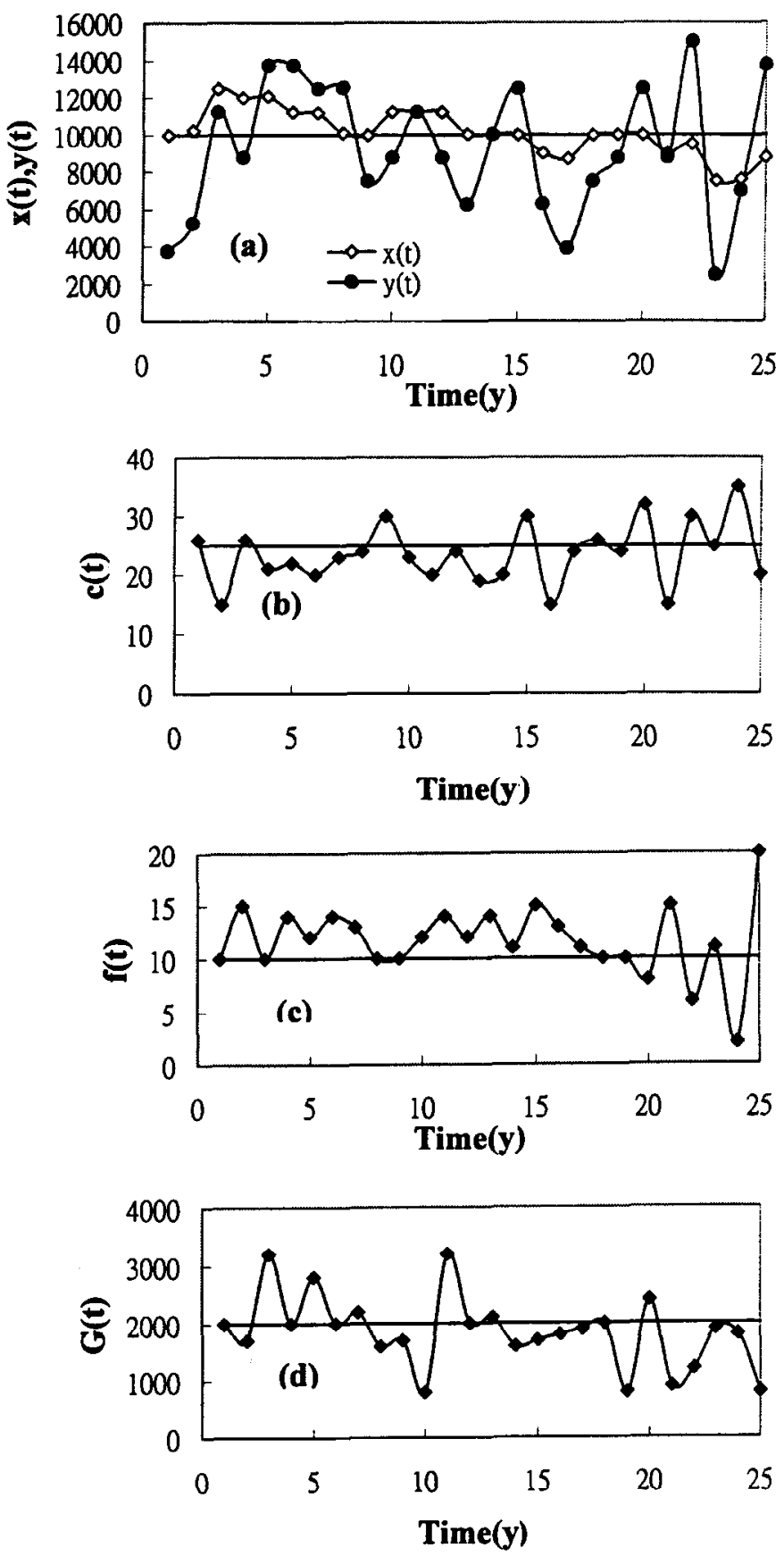

Fig. 6. Time profiles of (a) bioresource stock $x(t)$, (b) $\mathrm{Zn}$ concentration in abalone $c(t)$, (c) control effort $f(t)$, and (d) resource production function $\xi G(t)$ with desired equilibrium values shown as solid lines at $F=10, \mu=0.5$, and $\sigma_{\xi}{ }^{2}=0.25$. Measurements $y(t)$ are also shown with $x(t)$.

$\Delta c(t)$ given by Eq. 11, with $\Delta x(t)=\hat{x}(t \mid t)$ in which $\hat{x}(t \mid t)$ is given by Eq. 13 .

Figure 6 shows typical time-histories of bioresource stock $x(t)$, Zn concentration in Haliotis diversicolor supertexta $c(t)$, control effort $f(t)$, and resource 


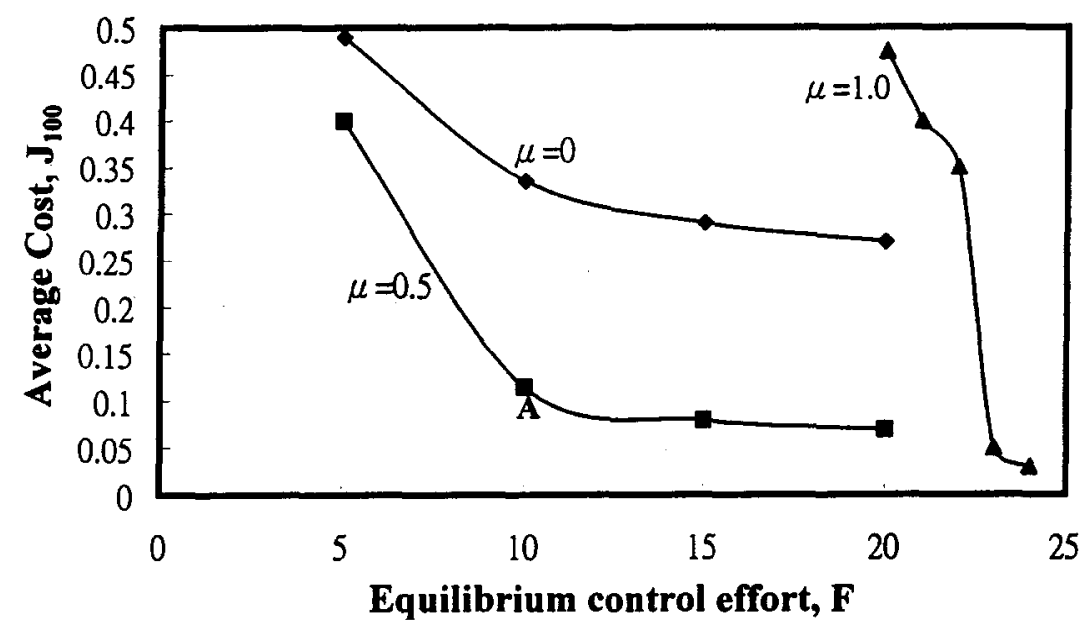

Fig. 7. Average cost $J_{100}$ against equilibrium $F$ with the model controlled by three different controllers in which point $A$ locates the simulation of Fig. 6 at $F=10, \mu=0.5$, and $\sigma_{\xi}^{2}=0.25$.

production function $\xi G(t)$, with desired equilibrium values shown as solid lines. Measurements $y(t)$ are also shown with the time-history of $x(t)$ at the operating point of $F=10, \mu=0.5$, and $\sigma_{\zeta}^{2}=0.25$.

The performance index was assessed by using the averaged sum $J_{T}$ in Eq. 8, with $\mu=0.5$ to represent equal weighting on fluctuations in $c$ and $f$. A simulation time $T$ of $100 \mathrm{y}$ was used to give the average costs of $J_{100}$. The performance of different controllers was compared by using the same sequence of computergenerated pseudo-random numbers to give the same sequence of disturbances and noises in each of the comparative simulations. Figure 7 shows some typical average costs $J_{100}$ from simulating control over periods of $100 \mathrm{y}$ with different desired equilibrium operating points, specified in terms of $F$, and with three different controllers. The suboptimal controller $\mu=0.5$ clearly gives the best control (smaller $J_{100}$ ) over the range of equilibrium $F$. The point $A$ in Fig. 7 locates the simulation of Fig. 6 at $\mu=0.5, F=10$, and $\sigma_{\xi}{ }^{2}=0.25$.

Suboptimal controllers designed with extreme values of the tuning parameter $\mu$ are the constant effort controller $\mu=0$, which is never as good as the $\mu=0.5$ controller, and the constant pollutant concentration controller $\mu=1$.

Figure 7 also indicates that costs escalate with increasing $F$ and with increasing $\mu$, as predicted by the discussion of stability analysis. The suboptimal feedback controller, with $\mu$ tuned in mid-range between the extremes of 0 and 1 , gives performance having timehistories which are the most likely to be acceptable.

\section{CONCLUSIONS}

This study investigated bioresource management in a polluted ecosystem from the viewpoint of feedback control engineering in the presence of uncertainty. By considering one particular problem regarding the $\mathrm{Zn}$ bioaccumulation in aquaculture abalone Haliotis diversicolor supertexta, it shows that engineering methodology can be used to illustrate problems in managing the harvest bioresource organisms in a polluted ecosystem. The chosen control problem was that of regulating a single trace metal concentration to a specified equilibrium state perturbed by random fluctuations in the annual recruitment of molluskan shellfish.

The stability analysis shows that the tuning parameter $\mu$ can serve as an indicator to reflect when a feedback control is required. If a certain high value of resource stocks is attempted, $\mu$ will approach 0 and a constant control effort, i.e., a feedback control is required. Simulations confirm that the designed feedback controller, when suitably tuned, gives satisfactory suboptimal control of the fully nonlinear single-state bioresource management model. Numerical results from the simulations show how the optimal choice of tuning parameter $\mu$ and the resulting costs vary with desired equilibrium state.

The resulting control laws include strategies of constant pollutant concentration in the biomass of the resource and constant control effort, both of which are incentives for the ecosystem managers. Numerical data are obtained which could guide the design of a controller minimizing fluctuations about a chosen 
equilibrium state. It is believed that the paper indicates how control engineering can contribute to solving problems of managing bioresources in a polluted ecosystem.

Acknowledgment-The author wishes to acknowledge the financial support of the National Science Council of Republic of China under Grant NSC 88-2621-B-002-005.

\section{REFERENCES}

Anderson, D.A. Optimal exploitation strategies for an animal population in a Markovian environment: $A$ theory and an example. Ecology 56: 1281-1297; 1975.

Aoki, M. Optimal control and system theory in dynamic economic analysis. Amsterdam: North-Holland Pub.; 1976.

Barnthouse, L.W. Population-level effects. In: Suter, G.W. II, ed. Ecological risk assessment. Chelsea, MI: Lewis Pub.; 1993: 247274.

Clark, C.W. Mathematical bioeconomics: The optimal management of renewable resources. $2^{\text {nd }}$ ed. New York, NY: John Wiley \& Sons; 1990.

Conory, P.T.; Hunt, J.W.; Anderson, B.S. Validation of a shortterm toxicity test endpoint by comparison with longer-term effects on larval red abalone Haliotis rufescens. Environ. Toxicol. Chem. 15: 1245-1250; 1996.

Edwards, C.A.; Grove, T.L.; Harwood, R.R.; Colfer, C.J.P. The role of agroecology: $\Lambda \mathrm{n}$ integrated farming systems in agricultural sustainability. Agric. Ecosyst. Environ. 46: 99-121; 1993.

Emlen, J.M.; Pikitch, E.K. Animal population dynamics: Identification of critical components. Ecol. Model. 44: 253-273; 1989.
Goh, B.S. Management and analysis of biological populations. Amsterdam: Elsevier; 1980.

Hines, W.W.; Montgomery, D.C. Probability and statistics in engineering and management science. New York, NY: John Wiley \& Sons; 1982.

Jocobs, O.L.R. Introduction to control theory. Oxford: Oxford University Press; 1993.

Liao, C.M.; Lin, W.Z. An optimal feedback control strategy for waste disposal management in agroecosystems. Appl. Math. Model. 21: 165-174; 1997.

Lin, M.C.; Liao, C.M. ${ }^{65} \mathrm{Zn}$ (II) accumulation in soft tissue and shell of abalone Haliotis diversicolor supertexta via the alga Gracilaria tenuistiptata var. liui and the ambient water. Aquaculture; 1999.

Mangel, M. Decision and control in uncertain resource systems. New York, NY: Academic Press; 1985.

Murray, J.D. Mathematical biology. New York, NY: SpringerVerlag; 1989.

Polderman, J.W.; Willems, J.C. Introduction to mathematical systems theory. New York, NY: Springer-Verlag; 1998.

Ritterhoff, J.; Zauke, G.-P. Bioaccumulation of trace metals in Greenland Sea copepod and amphipod collectives on board ship: Verification of toxicokinetic model parameters. Aquat. Toxicol. 40: 63-78; 1997.

Thomas, D.M.; Snell, T.W.; Jaffer, S.M. A control problem in a polluted environment. Math. Biosci. 136: 139-163; 1996.

Tiwari, S.; Ramaswamy, R.; Rao, J.S. Adaptive control in a resource management model. Ecol. Model. 84: 53-62; 1996.

Walters, C. Adaptive management of renewable resources. New York, NY: Macmillan; 1986.

Williams, B.K. Adaptive optimization and the harvest of biological populations. Math. Biosci. 136: 1-20; 1996. 